Z. klin. Chem. u. klin. Biochem.

8. Jg., S. $374-378$, Juli 1970

\title{
Das elektronenmikroskopische Bild der Rattenaorta bei Mg-Mangel
}

\author{
Von H.-J. Merker und Th. GüNTher \\ Aus dem II. Anatomischen Institut der Freien Universität Berlin (Direktor: Prof. Dr. W. Schwarq) \\ und dem Pby'siologisch-chemischen Institut der Freien Universität Berlin (Direktor: Prof. Dr. Dr. E. Scbütte)
}

(Eingegangen am 25. März 1970)

Nach 38 tägiger Verfütterung einer Mg-armen Nahrung lassen sich an den glatten Muskelzellen der Rattenaorta elektronenmikroskopisch Mitochondrienschwellung, getinge Vermehrung größerer Vesikel (1000-2000 $\AA$ ) und Lysosomen-ähnliche Einschlüsse, unregelmäßiger Verlauf der Zelloberfläche und eine Vermehrung des rauhen endoplasmatischen Retikulums darstellen. Die Morphologie der Fibrocyten ändert sich nicht, die Endothelzellen zeigen neben einer Mitochondrienschwellung und Vermehrung von Vakuolen eine Erweiterung der Interzellularspalte ohne Veränderungen an den tight junctions. Die Dichte der elastischen Fasern und die Zahl ihrer Filamente nehmen ab. Die Einlagerung von Ca-Kristallen beginnt vorwiegend an der Peripherie der elastischen Fasern. Zehn Tage nach Wiederverfütterung einer $\mathrm{Mg}$-reichen $\mathrm{Nahrung}$ sind die Veränderungen bis auf die Kalkeinlagerungen wieder verschwunden.

\section{The electron microscopic structure of rat aorta in $M g$ deficiency}

After 38 days on a Mg-deficient diet, the electron microscope picture of the smooth muscle of rat aorta shows mitochondrial swelling, a slight increase in the large vesicles $(1000-2000 \AA)$ and lysosome-like inclusions, an irregular cell surface and an increase in the rough endoplasmic reticulum. The morphology of the fibrocytes is unchanged, the endothelial cells show mitochondrial swelling and increase of vacuoles, together with an expansion of the intercellular spaces, with no changes in the tight junctions. The thickness of the elastic fibres and the number of their filaments is decreased. The deposition of calcareous crystals starts chiefly at the periphery of the elastic fibres. Ten days after the reintroduction of a Mg-rich diet, the above changes can no longer be observed, except for the presence of the calcareous deposits.

Bei $\mathrm{Mg}$-arm ernährten Tieren treten u. a. an den Arterien Arteriosklerose-ähnliche Veränderungen auf (1, 2, 3). Die dabei ablaufenden Vorgänge, die Reihenfolge ihrer Entstehung sowie ihre Reparation nach Wiederverfütterung einer $\mathrm{Mg}$-reichen Diät wurden bisher noch nicht elektronenmikroskopisch untersucht und sollen in der vorliegenden Arbeit mitgeteilt werden.

\section{Material und Methode}

Die Tiere wurden in der bereits beschriebenen Weise behandelt $(4,5)$. Die Aorta thoracalis wurde am 4., 6., 11., 14., 22. und 38. Tage nach Beginn der $\mathrm{Mg}$-armen Fütterung oder 1, 3, bzw. 10 Tage nach Wiederverfütterung einer $\mathrm{Mg}$-reichen $\mathrm{Diät}$, zu deren Beginn $2 \mathrm{~m} / 0,1 \mathrm{M} \mathrm{MgCl}_{2}$-s. c. gegeben wurde, in Äthernarkose entnommen und mit einer modifizierten Karnovsky-Lösung aus $3 \%$ Glutaraldehyd und 3\% Paraformaldehyd in $0,1 \mathrm{M}$ Phosphatpuffer $(\mathrm{pH} \mathrm{7,2)}$ fixiert. Nach erneuter Fixierung in gepufferter 1 proz. $\mathrm{OsO}_{4}$-Lösung wurden die Aorten in dünne Scheiben zerschnitten und in Micropal eingebettet. Zur genauen Orientierung wurden Semidickschnitte hergestellt und in 50proz. wäßr. GiemsaLösung gefärbt. Eine gute lichtmikroskopische Beurteilung der Schnittrichtung ist dadurch möglich. Schnitte: LKB-Ultratome. Aufnahmen: Siemens Elmiskope I und Ia. Nachkontrastierung: Uranylacetat/Bleicitrat.

\section{Befunde}

Nach 38 tägiger Verfütterung einer Mg-armen Nahrung bestehen deutliche Veränderungen an den verschiedenen Strukturen der Rattenaorta. Wie bei anderen Zellarten sind auch die Mitochondrien der glatten Muskelzellen in der Aorta geschwollen (Abb. 1). Dabei geht etwas Matrix verloren, die Länge und Zahl der Cristae wird geringer und die Mitochondrien sind abgerundet. Daneben kann eine Vermehrung von größeren (1000 bis

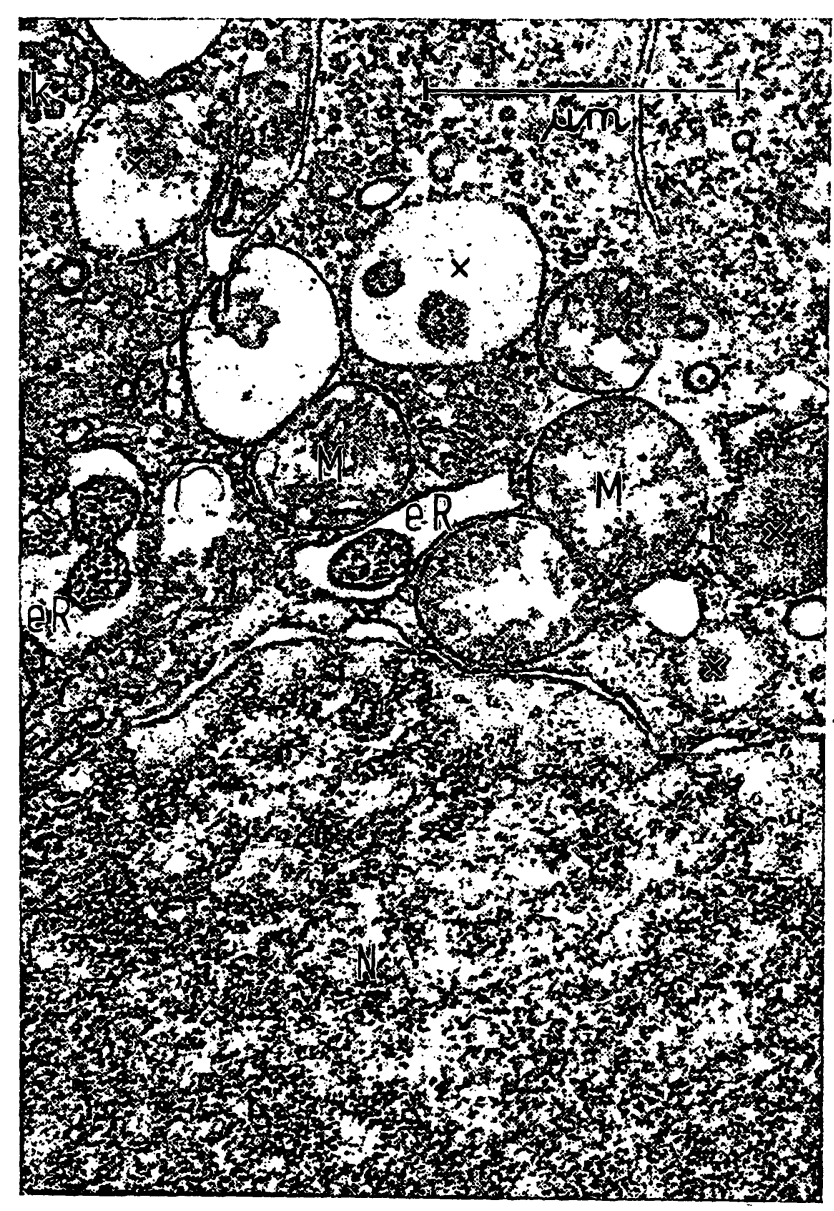

Abb. 1

Ausschnitt aus einer glatten Muskelzelle der Rattenaorta (22 Tage Mg-Mangel) mit Vakuolen und Granula $(x)$, leicht geschwollenen Mitochondrien $(M)$ und erweiterten Hohlräumen des rauhen eR.
$\mathrm{K}=$ Bündel kollagener Fibrillen an der Zellmembran. $\mathrm{N}=$ Nukleus. Vergrößerung 1:30000 
$2000 \AA)$ Vesikeln und elektronendichten etwa 0,2 bis $0,3 \mu \mathrm{m}$ großen Einschlüssen im Cytoplasma beobachtet werden (Abb. 1). Diese Einschlüsse enthalten granuläres und membranartiges Material. Morphologisch entsprechen sie also Lysosomen. Die Hohlräume des rauhen endoplasmatischen Retikulums (eR) sind deutlich vermehrt (Abb. 2). Diese Zunahme wird bei der Ausbildung des Mg-Mangels zuerst an den Myofilamentfreien Kernpolen sichtbar. Rauhes eR wird aber auch in der Zellperipherie gefunden, wo es bei normalen glatten Muskelzellen nur selten vorkommt. Diese Zellen weisen bei Mg-Mangel außerdem einen unregelmäßigen Verlauf ihrer Zelloberfläche, d.h. eine Oberflächenvergrößerung auf. Sie zeigen im Mg-Mangel Einstülpungen sowie kleine, plumpe und unregelmäßig geformte Fortsätze. Die große Zahl der mikropinocytotischen Vesikel, die für glatte Muskelzellen typisch ist, ist dabei jedoch verringert (Abb. 3).

In den Endothelzellen der Rattenaorta können im MgMangel ebenfalls leichte Mitochondrienschwellungen und eine Vermehrung von Vakuolen beobachtet werden. Stellenweise sind die interzellulären Räume stark erweitert. Bereits in der normalen Aorta kommen neben Verzahnungen und dem typischen relativ gradlinigen Interzellularraum von etwa $200 \AA$ Breite kleinere Erwei-

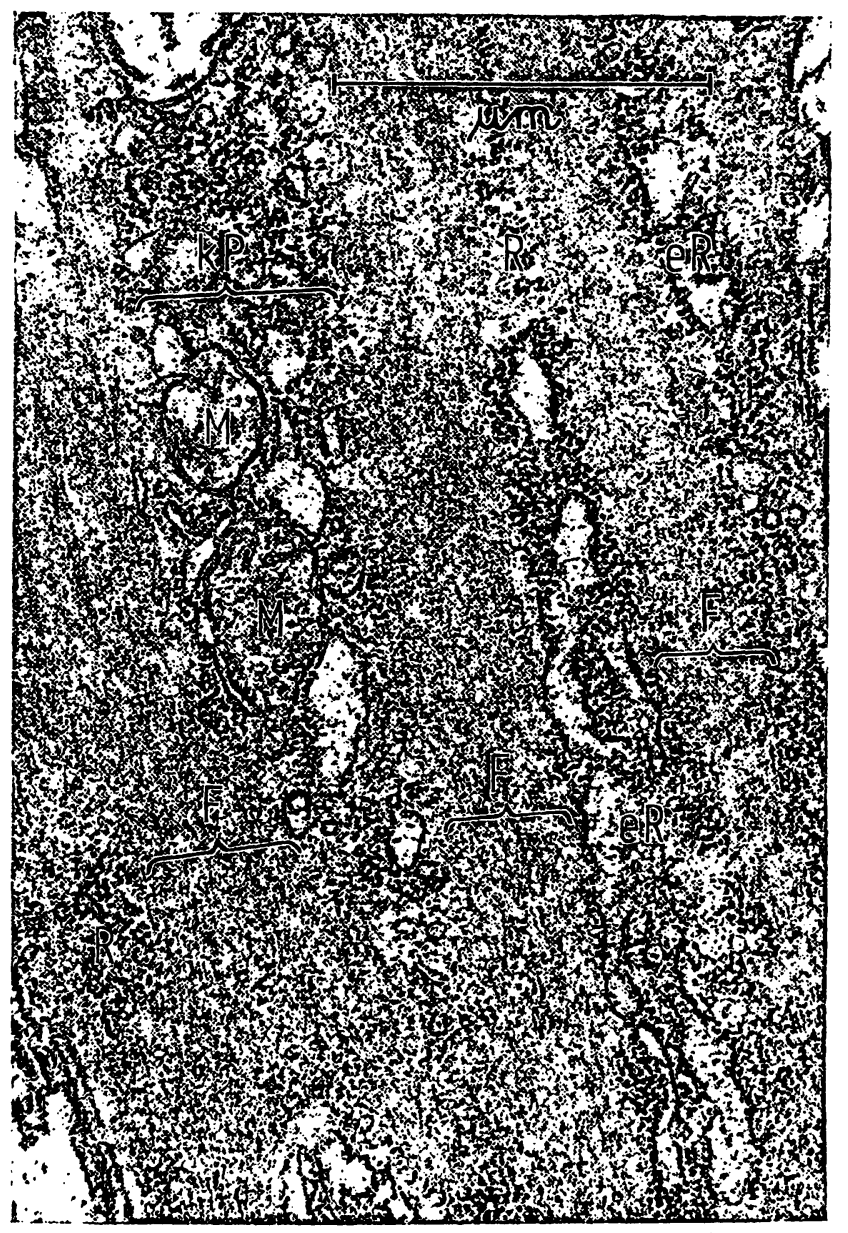

Abb. 2

Ausschnitt aus einer glatten Muskelzelle der Rattenaorta (38. Tage Mg-Mangel) mit vermehrtem rauhen eR und Ribosomen (R) zwischen
den Myofilamentbündeln $(F)$ KP $=$ Kernpol. $M=$ Mitochondrien. den Myofilamentbündeln (F). KP $=$ Kernpol.
Vergrößerung 1:40000 terungen vor. Im Mg-Mangel hat jedoch die Zahl und Größe dieser Erweiterungen deutlich zugenommen. Die zum Lumen hin gelegenen Abdichtungen des interzellulären Spaltes, die tight junctions oder zonulae occludentes, stellen sich unverändert dar (Abb. 4). An den Fibrocyten der Aorta waren keine wesentlichen Veränderungen zu erfassen.

Im extrazellulären Ralum sind an der Dicke, Packungsdichte und am Querstreifungsmuster der kollagenen Fibrillen keine Abweichungen von den Kontrolltieren nachzuweisen.

Deutliche Abweichungen vom normalen Bild zeigen dagegen die elastischen Fasern. In der normalen Aorta bestehen sie aus unregelmäßig verlaufenden, in der Peripherie angereicherten 50-100 \& dicken Filamenten, sowie einer homogenen Grundsubstanz von mittlerer Elektronendichte. Nach Verabreichung einer Mg-Mangeldiät verringert sich die $\mathrm{Zahl}$ der Filamente und die Elektronendichte der Matrix nimmt ab. Die elastischen Fasern bestehen dann nur noch aus zarten, kaum sichtbaren homogenen Bändern (Abb. 5).

An diesen veränderten elastischen Fasern der Aorta bei $\mathrm{Mg}$-Mangel finden sich Ca-Ablagerungen. Häufig beginnt die Verkalkung in der Peripherie der elastischen Fasern und schreitet zum Zentrum hin fort. Feine nadel-

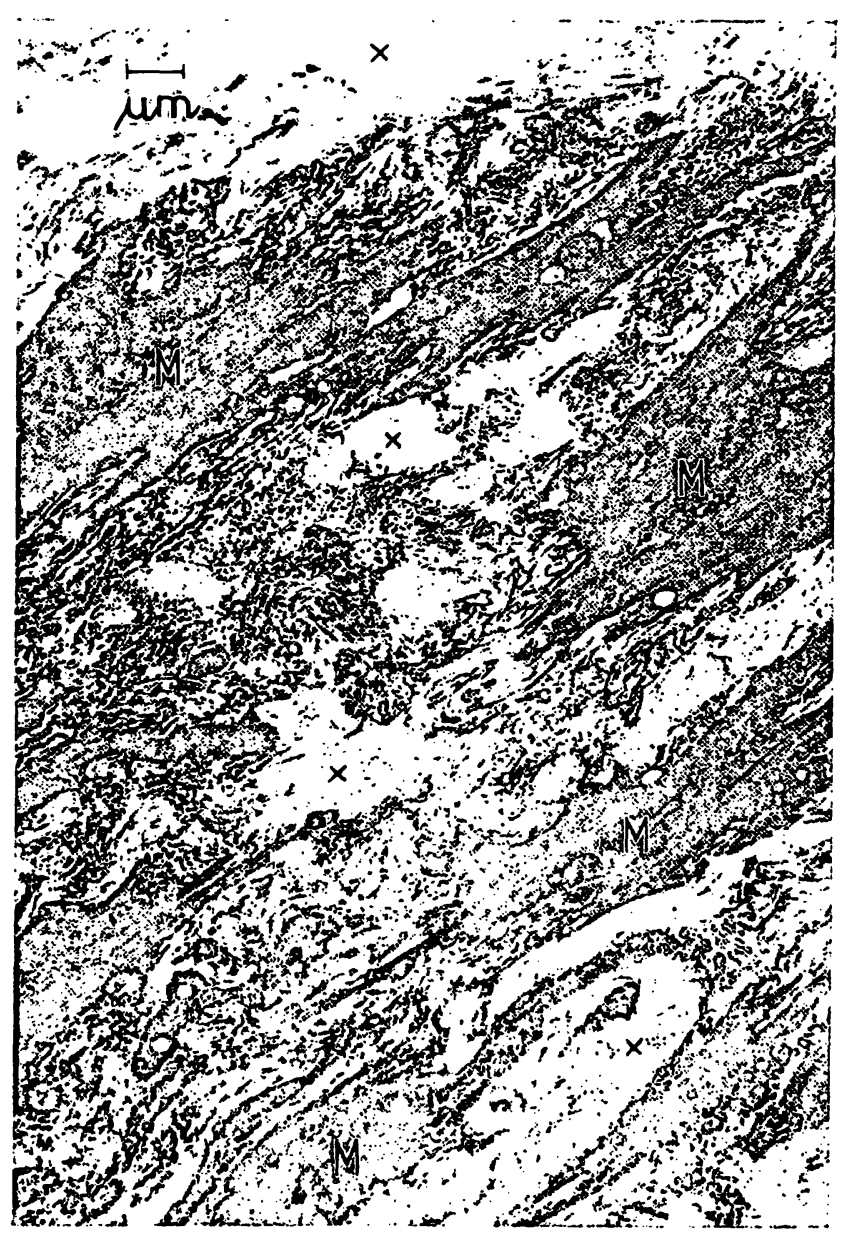

Abb. 3

Ubersichtsbild von angesclnittenen glatten Muskelzellen der Raiten-

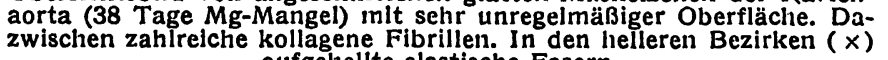
aufgehellte elastische Fasern. 


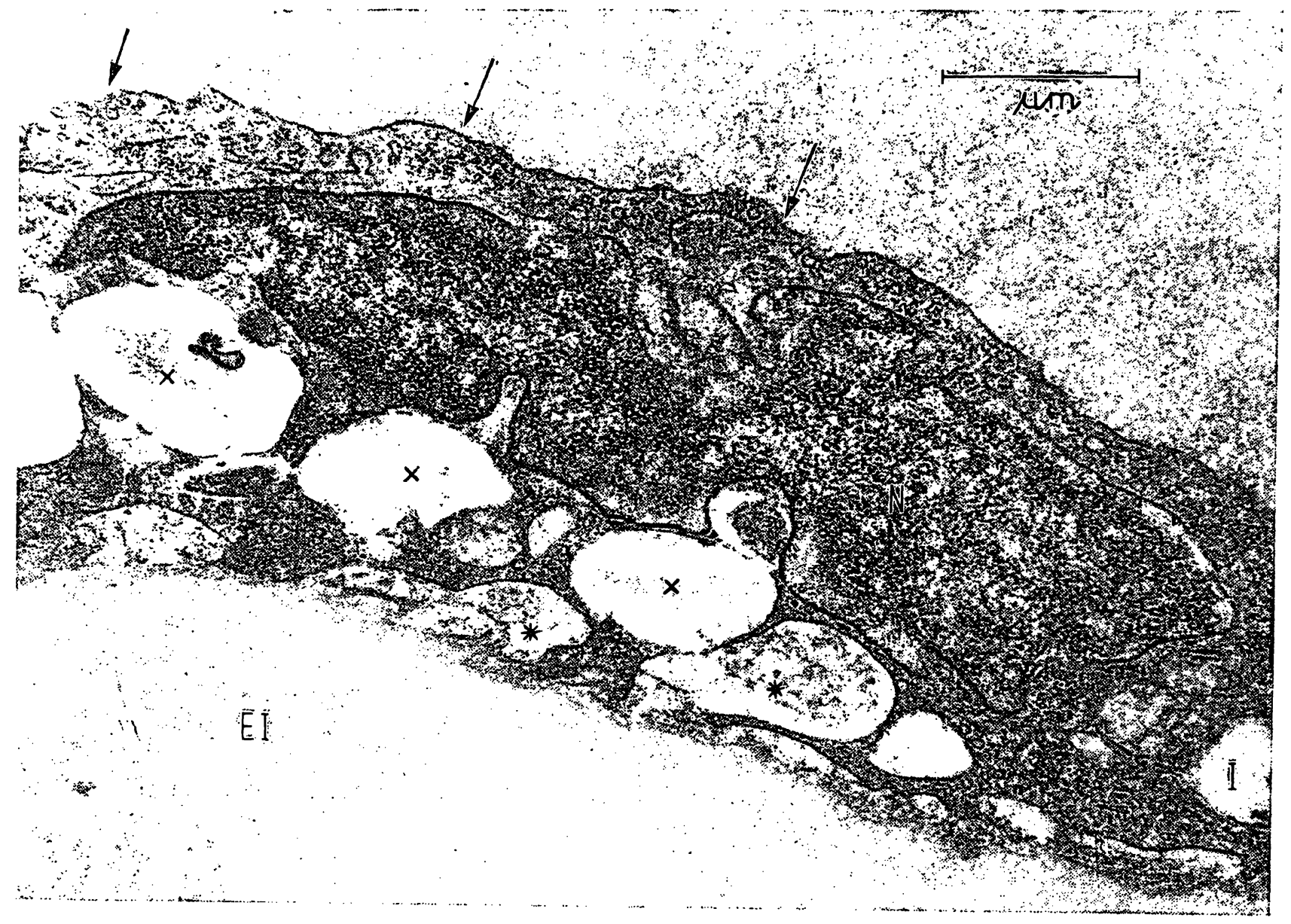

Abb. 4

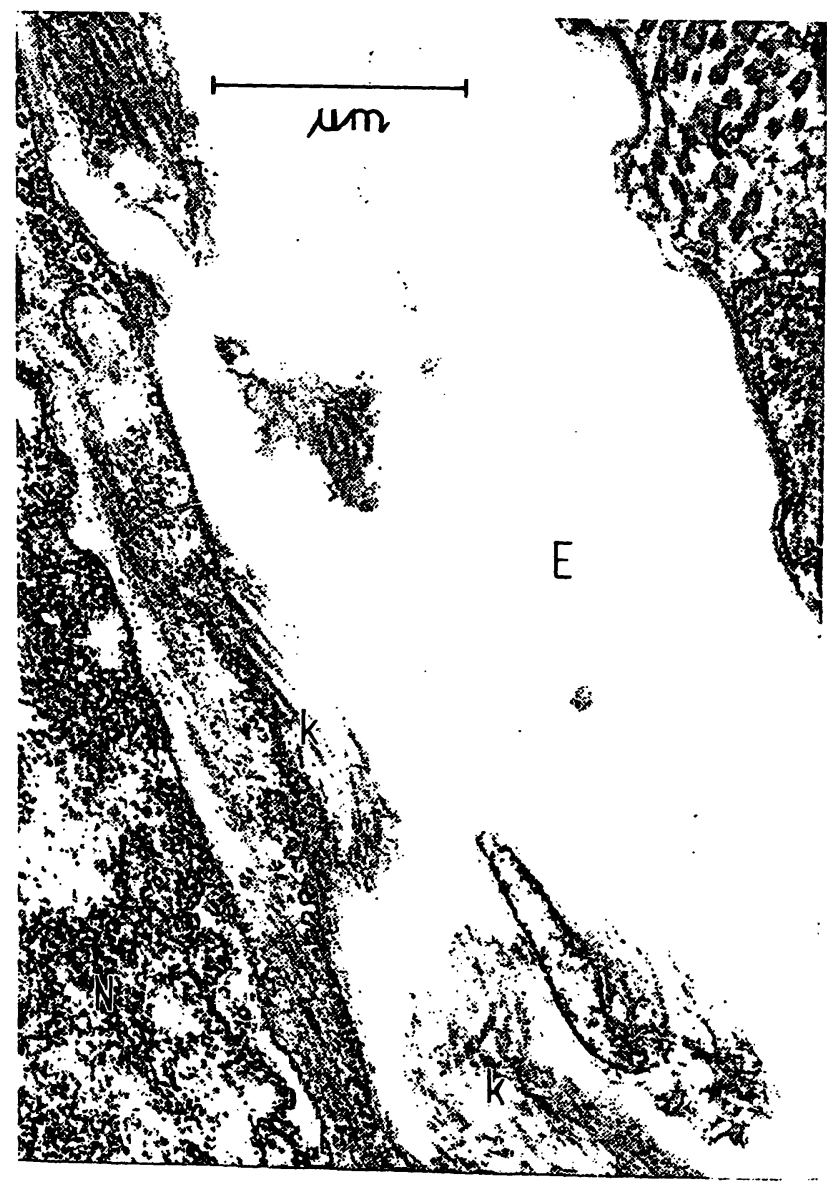

Abb. 5

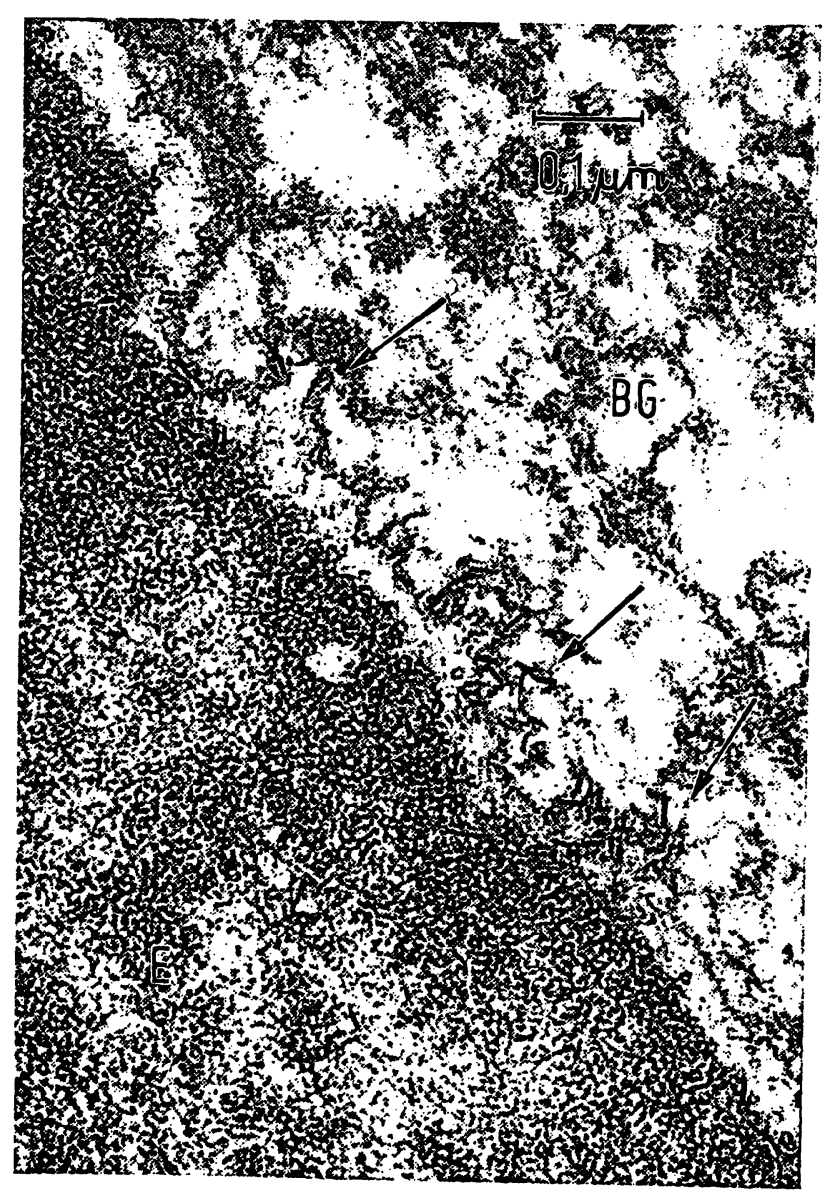

Abb. 6 


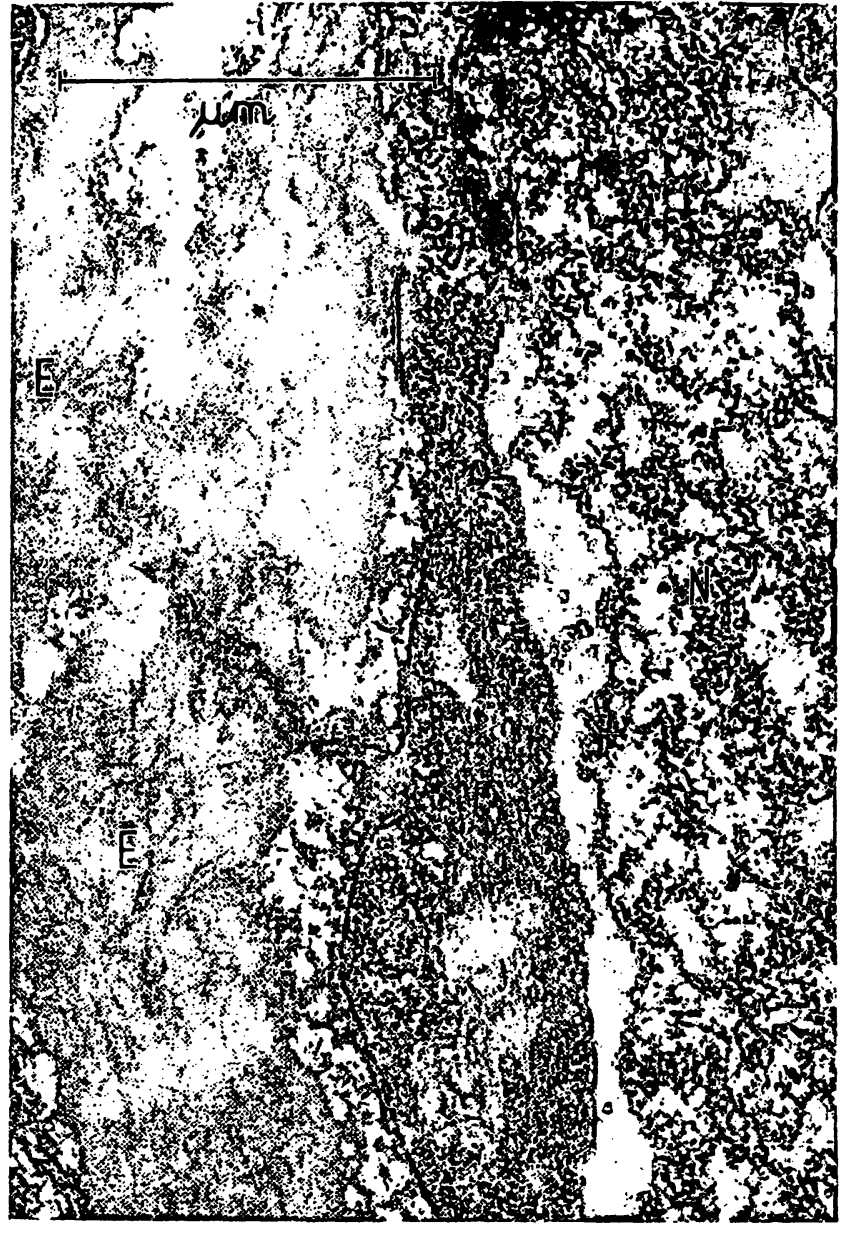

Abb. 7

Elastische Fasern (E) in einer Rattenaorta 10 Tage nach Wiederverfütterung einer $\mathrm{Mg}$-reichen Nahrung. Elastische Grundsubstanz und Filamente wieder vorhanden. $\mathbf{N}=$ Nukleus einer glatten Muskelzelle Vergrößerung 1:35000

oder lanzettförmige Kristalle mit einer Breite von 40 bis $100 \AA$ und einer Länge von $600 \AA$ lagern sich regellos oder senkrecht zur Faserverlaufsrichtung ein (Abb. 6). Daneben können aber schon anfangs einige drusenförmige Ca-Ablagerungen im Zentrum der Fasern beobachtet werden, deren Größe mit der Dauer der Mgarmen Ernährung zunimmt. - Neben diesen typischen Ablagerungen in den elastischen Fasern kommen noch drusenförmige Plaques an anderen Stellen des intraund extrazellulären Raumes vor. Sie entsprechen in ihrer Morphologie den bereits beschriebenen $\mathrm{Ca}$-Ablagerungen in anderen Geweben (5).

Abb. 4

Entdothelzelle der Rattenaorta (22, Tage Mg-Mangel) mit starker Vesikulation $(\downarrow)$ an der Lumenseite, Vakuolen basal $(x)$, tiefe Einstülpungen (*) und ein erweiterter Interzellularspalt (I). EI I' = Elastica interna. $N=$ Nukleus
Vergrößerung $1: 25000$

Abb. 5

Veränderte elastische Faser (E) in einer Rattenaorta (38 Tage $\mathrm{Mg}$ Mangel). Fehlende elastische Grundsubstanz und Verringerung der Filamentzahl. $\mathrm{K}=$ kollagene Fibrillen, $\mathrm{N}=$ Nukleus einer glatten

Vergrößerung 1:25000

Abb. 6

Rattenoarta (22 Tage Mg-Mangel). Beginnende Verkalkung in der Peripherie einer elastischen Faser (E). Feine längsprismatische Kristalle ( $\downarrow)$ mit unregelmäßigem Verlauf. $B G=$ Bindegewebige Interzellularsubstanz

Vergrößerung 1:105 000
Die beschriebenen Veränderungen in der Aorta treten mit untcrschiedlicher Geschwindigkeit auf. Dic Mitochondrienschwellung und die Vermehrung des rauhen $\mathrm{cR}$ ist bereits 4 Tage nach der Verfütterung einer $\mathrm{Mg}$ armen Diät nachweisbar, die Zerklüftung der Zelloberfläche am 6. Tag, die Veränderungen an den elastischen Fasern und an den Endothclzellen am 11. Tag, das vermehrte Auftreten von Lysosomen und Vakuolen am 14. Tag und schließlich deutliche Verkalkungen am Beginn der 3. Woche.

Nach Wiederverfütterung einer Mg-reichen Diät sind die Veränderungen an den Mitochondrien, im Endothel, an der Zelloberfläche, die Vermehrung der Lysosomen, der Vakuolen und des rauhen $\mathrm{eR}$, sowie die Aufhellung der elastischen Fasern nach 10 Tagen fast oder vollständig verschwunden (Abb. 7). Die Verkalkungen zeigen dagegen in diesem Zeitraum keine Rückbildungen.

\section{Diskussion}

Während die Mitochondrienschwellung, die Vakuolenund die Oberflächenzunahme auch in anderen Geweben gefunden und bereits an anderer Stelle (5) diskutiert wurden, stellen die Veränderungen der elastischen Fasern, des Endothels und der glatten Muskelzellen sowie der Verkalkungsmodus wahrscheinlich eine besondere Wirkung des Mg-Mangels auf die Arterienwand dar, denn bei den bisherigen morphologischen Untersuchungen der $\mathrm{Mg}$-Mangel-Tiere zeigten sich in anderen Organen keine deutlichen Veränderungen an den entsprechenden Strukturen. Zur Erklärung der Sonderstellung der glatten Muskelzellen in der Aorta kann auf ihre von anderen glatten Muskelzellen abweichende Reifung hingewiesen werden. Sie bilden zunächst als Fibroblasten die bindegewebige Grundsubstanz der Gefäßwand, hauptsächlich die elastischen Fasern. Erst dann differenzieren sie sich zu glatten Muskelzellen, die jedoch die Fähigkeit zur Elastinsynthese beibehalten (6). Die elastischen Fasern der Aorta zeichnen sich gegenüber anderen elastischen Fasern durch eine besondere Textur im Sinne einer Querverspannung zwischen den Muskellamellen aus. Es besteht hier eine ausgeprägte elastisch-muskuläre Funktionseinheit (7). Bei den Endothelzellen der großen Gefäße schließlich ist die mechanische Beanspruchung hervorzuheben, die bevorzugt zu morphologischen Veränderungen führen kann.

Die elektronenoptisch sichtbare Aufhellung des Elastins läßt sich mit einer Abnahme der Elastinmenge erklären. BrITTON und Mitarbeiter (8) fanden nach vierwöchiger Mg-armer Ernährung (100 ppm Mg) eine Abnahme des Elastingehaltes in der Rattenaorta von $20 \%$, wobei der Einbau von 14-C-Glycin in Elastin um 60\% verringert war. Das zeigt, daß im Mg-Mangel die Biosynthese von Elastin vermindert wird. Im elelktronenmikroskopischen Bild besteht die elastische Faser aus einer homogenen Grundsubstanz mittlerer Elektronendichte, in die 50 bis $100 \AA$ dicke Filamente eingelagert sind. Die homogene Matrix enthält das Elastomucin, während dic Filamente aus Protein bestehen. Nach unseren elektronenmikroskopischen Bildern sind beide Bestandteile im $\mathrm{Mg}$ - 
Mangel deutlich verringert. Am Kollagen, das von den gleichen Zellen gebildet wird, waren keine Änderungen sichtbar. Entweder ist der turn-over des Kollagens wesentlich langsamer als der des Elastins oder im MgMangel ist die Elastinsynthese spezifisch betroffen. Für die Elastinsynthese kommen neben den Fibroblasten der Adventitia vorwiegend die glatten Muskelzellen der Aorta in Frage. Die Fibroblasten erscheinen unverändert, die Muskelzellen dagegen waren zu Fibroblastenähnlichen Zellen mit stark ausgeprägten rauhem endoplasmatischem Retikulum umgewandelt. Da die Elastinsynthese trotz einer Vermehrung des tauhen endoplasmatischen Retikulums in den glatten Muskelzellen gestört ist, kann man auf eine qualitative Änderung ihrer Syntheseleistung schließen.

Die elektronenmikroskopisch sichtbare Vermehrung des rauhen $\mathrm{e}$ der glatten Muskelzellen spricht für eine höhere Stoffwechselaktivität in diesen Zellen, die bei der Arteriosklerose nach Cholesterinfütterung direkt gemessen wurde, deren Bedeutung aber unklar ist $(9,10,11)$.

Die extrazellulären $\mathrm{Ca}$-Niederschläge waren fast ausschließlich an den Elastinfasern lokalisiert. In ${ }^{45} \mathrm{Ca}$ Einbau-Versuchen (12) mit isolierten Aorten und Stükken aus dem Lig. nuchae (Elastin) unter Verwendung verschiedener Inhibitoren ergab sich, daß die Verkalkung dieser Gewebe vom Stoffwechsel unabhängig ist und durch die Struktur des Elastins (Vorhandensein freier Amino- und Sulfhydrylgruppen) bzw. der organischen Matrix bedingt ist. Es ist daher wahrscheinlich, $\mathrm{daB}$ im Mg-Mangel das Elastin bzw. die organische Matrix der Aorta nicht nur quantitativ, sondern auch qualitativ verändert wird und sich dadurch Ca-Niederschläge bilden können. Hierfür spricht auch, daß Verkalkungen erst dann auftreten (in unseren Versuchen nach 3 Wochen), wenn die extrazelluläre Mg-Konzentration schon längere Zeit abgesunken ist (Abb. 8). Bei extrem Mg-armer Diät, wenn die Serum-Mg-Konzentration schneller absinkt, können die Tiere schon vor dem Auftreten von Verkalkungen sterben (8). An Hunden schließlich wurden im $\mathrm{Mg}$-Mangel $\mathrm{Ca}$-Ablagerungen in den Arterien bei nur wenig erniedrigter Mg-Konzentration gefunden (13). Für die Bildung extrazellulärer Ca-Niederschläge scheint daher eine Veränderung des $\mathrm{Ca} / \mathrm{Mg}$-Verhältnisses in der extrazellulären Flüssigkeit beim Mg-Mangel nicht entscheidend zu sein.

Die zusätzlich auftretende Verkalkung in den glatten Muskelzellen entspricht den früher beschriebenen intra-

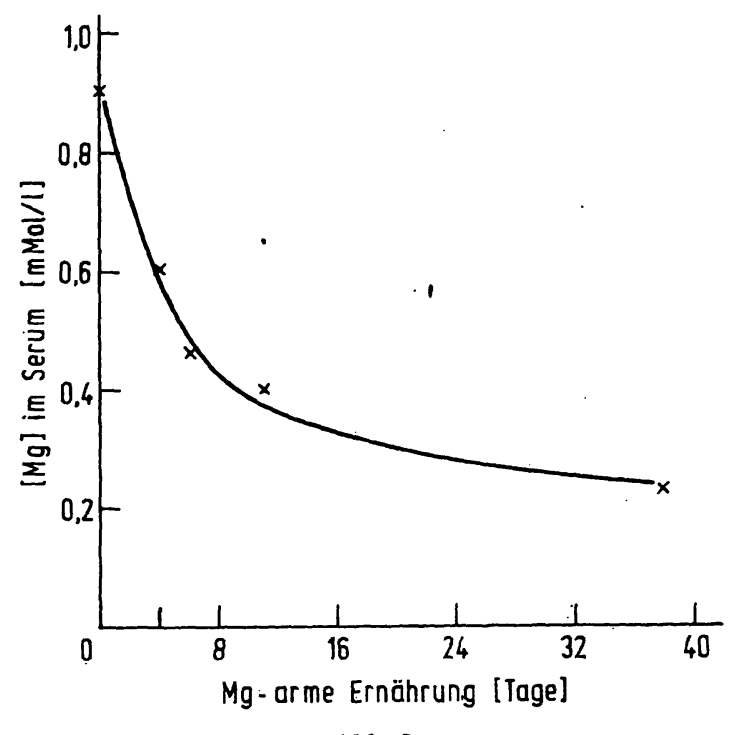

Abb. 8

Mg-Konzentration im Serum bei Mg-armer Ernährung. Das Serum wurde mit 10 proz. Trichloressigsäure, die $0,1 \%$ La enthielt, enteiweißt und im Atomabsorptionsspektrophotometer (M4QIII-FA2, Fa. Zeiss) gemessen

zellulären Ca-Ablagerungen in anderen Zellen (5). $\mathrm{Da}$ Ca durch die Zellmembran permeiert, könnte die intrazelluläre Verkalkung durch die erhöhte $\mathrm{Ca}$ - und erniedrigte $\mathrm{Mg}$-Konzentration in der extrazellulären Flüssigkeit bedingt sein.

Die Endothelzellen der Aorta reagieren mit Vakuolenbildung und Erweiterung der Interzellularräume. Eine Schädigung der zonulae occludentes (tight junction) war jedoch nicht nachzuweisen. Über eine Permeabilitätserhöhung der Endothelschicht kann daher nichts gesagt werden, obgleich bei der Entstehung arteriosklerotischer Veränderungen von verschiedenen Autoren (7, 14-16) ein vermehrter Einstrom von Blutplasma in die Aortenwand angenommen wird.

Die charakteristische Veränderung der Rattenaorta im Mg-Mangel ist die Aufhellung der elastischen Fasern und deren Verkalkung. Bei anderen Arterioskleroseformen dagegen fragmentieren die elastischen Fasern, kollagene Fibrillen werden neu gebildet, histiocytäre Zellelemente sind vermehrt vorhanden und fast alle Zelltypen beladen sich mit Lipideinschlüssen. Man kann aus diesen Unterschieden schließen, $\mathrm{da}$ die anderen Arterioskleroseformen nicht durch $\mathrm{Mg}$-Mangel mitverursacht werden, obgleich $\mathrm{Mg}$ mit $\mathrm{Ca}$ bei der Bildung von $\mathrm{Ca}$-Niederschlägen konkurrieren und somit deren Entstehung hemmen kann.

\section{Literatur}

1. Moore, L. A., E. T. Hallmann und L. B. Sholl, Arch. Path., Chicago 26, 820 (1938). - 2. Unglaub, I., I. SyllmRapoport und I. Strassburger, Virchows Arch. path. Anat. 332, 122 (1959). 3. Morris, M. L., jun., W. R. Featherston, P. H. Philips und S. T. McNult, J. Nutrit. 79, 437 (1963). - 4. GüNTHER, TH., diese Z. 8, 65 (1970). - 5. MERKER, H.-J. und Th. GüNTHER, diese Z. 8, 71 (1970).- - 6. Schwarz, W., Verh. Anat. Ges. 56, 308 (1959). - 7. Duguid, J. B., J. Path. Bact. 60, 57 (1949). 8. Britton, W. M. und E. L. R. Stokstad, Fed. Proc. $28 ; 562$
(1969). - 9. Scotr, R. F. und E. S. Morrison, Fed. Proc. 27, 576 (1968). - 10. Lee, K. T., D. N. Kimm und S. C. NAM, Fed. Proc. 27, 576 (1968). - 11. Jones, R. M. und K. T. LeE, Fed. Proc. 27, 575 (1968). - 12. Schiffmann, E., G. R. Martin und B. A. CorCORAN, Arch. Biochem. Biophysics 107, 284 (1964). - 13. VITALE, J. J., E. E. Hellerstein, M. Nakamura und B. Lown, Circulation Res. 9, 387 (1961). - 14. IInZBACH, J., Verh. dtsch. Ges. Pathol. 41, 24 (1957). - 15. Bragdon, J. H., Circulation 4, 466 (1951). - 16. Bredr, H., Verh. dtsch. Ges. Pathol. 41, 11 (1957).

Prof. Dr. H.-J. Merker

II Anat. Inst. d. FU, 1 Berlin 33, Königin-Luise-Str. 15 